\title{
\begin{tabular}{l|l} 
Mibraries & DSpace@MIT
\end{tabular}
}

\author{
MIT Open Access Articles
}

\section{Two-photon 3-D mapping of ex vivo human skin endogenous fluorescence species based on fluorescence emission spectra}

The MIT Faculty has made this article openly available. Please share how this access benefits you. Your story matters.

Citation: Laiho, Lily H., Serge Pelet, Thomas M. Hancewicz, Peter D. Kaplan, and Peter T. C. So. "Two-Photon 3-D Mapping of Ex Vivo Human Skin Endogenous Fluorescence Species Based on Fluorescence Emission Spectra." Journal of Biomedical Optics 10, no. 2 (2005): 024016. (C) 2005 Society of Photo-Optical Instrumentation Engineers

As Published: http://dx.doi.org/10.1117/1.1891370

Publisher: SPIE

Persistent URL: http://hdl.handle.net/1721.1/87642

Version: Final published version: final published article, as it appeared in a journal, conference proceedings, or other formally published context

Terms of Use: Article is made available in accordance with the publisher's policy and may be subject to US copyright law. Please refer to the publisher's site for terms of use. 


\section{Two-photon 3-D mapping of ex vivo human skin endogenous fluorescence species based on fluorescence emission spectra}

\author{
Lily H. Laiho* \\ Serge Pelet \\ Massachusetts Institute of Technology \\ Department of Mechanical Engineering \\ 77 Massachusetts Avenue, NE47-276 \\ Cambridge, Massachusetts 02139 \\ Thomas M. Hancewicz \\ Peter D. Kaplan \\ Unilever Research and Development, Edgewater \\ Advanced Imaging and Measurement \\ 45 River Road \\ Edgewater, New Jersey 07020
}

Peter T. C. So

Massachusetts Institute of Technology

Department of Mechanical Engineering and

Division of Biological Engineering

77 Massachusetts Avenue, NE47-279

Cambridge, Massachusetts 02139

E-mail: ptso@mit.edu

\begin{abstract}
Spectral resolved tissue imaging has a broad range of biomedical applications such as the minimally invasive diagnosis of diseases and the study of wound healing and tissue engineering processes. Two-photon microscopy imaging of endogenous fluorescence has been shown to be a powerful method for the quantification of tissue structure and biochemistry. While two-photon excited autofluorescence is observed ubiquitously, the identities and distributions of endogenous fluorophores have not been completely characterized in most tissues. We develop an image-guided spectral analysis method to analyze the distribution of fluorophores in human skin from 3-D resolved two-photon images. We identify five factors that contribute to most of the luminescence signals from human skin. Luminescence species identified include tryptophan, $\mathrm{NAD}(\mathrm{P}) \mathrm{H}$, melanin, and elastin, which are autofluorescent, and collagen that contributes to a second harmonic signal. () 2005 Society of Photo-Optical Instrumentation Engineers. [DOI: 10.1117/1.1891370]
\end{abstract}

Keywords: spectroscopy; two-photon microscopy; second harmonic generation; autofluorescence; human skin.

Paper 04067 received May 4, 2004; revised manuscript received Oct. 21, 2004; accepted for publication Oct. 21, 2004; published online Mar. 30, 2005. This paper is a revision of a paper presented at the SPIE conference on Optical Diagnostics of Living Cells IV, Jan. 2001, San Jose, CA. The paper presented there appears (unrefereed) in SPIE Proceedings Vol. 4260.

\section{Introduction}

Endogenous fluorophores are responsible for tissue autofluorescence. Autofluorescence imaging can provide useful tissue structural and biochemical information without fixation and staining procedures, offering the opportunity to develop optical biopsy based on fluorescence contrast. Many endogenous fluorescent species can be effectively imaged using twophoton microscopy, which allows the imaging of tissue structures in three dimensions. The principles for two-photon excitation were developed by Goppert-Mayer. ${ }^{1}$ Two-photon excitation occurs when an electron is excited to a higher energy electronic state by the simultaneous absorption of two photons in the infrared wavelength. The two photons have half the energy of the single photon necessary in conventional fluorescence imaging. When the excited electron returns to the ground state, it emits a fluorescence photon. Since the cross section of two-photon processes is significantly less than in the one-photon case, and the two-photon fluorescence has a quadratic dependence on excitation power, the two-photon excitation only takes place in a localized subfemtoliter excitation volume at the focal point where there is a high concentration of photons. This localization of excitation provides

*Current affiliation: Univ. of Hawaii at Manoa, Dept. of Mechanical Engineering, 2540 Dole St., Holmes Hall 302, Honolulu, HI 96822, E-mail: laiho@hawaii.edu depth discrimination and allows us to obtain 3-D images in a laser-scanning microscope system, as was first demonstrated by Denk, Strickler, and Webb. ${ }^{2}$

Two-photon excitation has several important advantages over one-photon techniques, such as confocal microscopy, for imaging tissue autofluorescence. ${ }^{3}$ Since fluorescence excitation only occurs in a small region, this reduces photodamage and photobleaching, allowing the study of living specimens. Furthermore, tissue endogenous fluorophores have absorption in the ultraviolet (UV) spectral range. Since UV radiation is strongly absorbed and scattered by the tissue, UV microscopic imaging of tissue typically has a very short penetration depth. The use of two-photon methods allows the excitation of UV fluorophores in the infrared wavelength region, where the tissue scattering and absorption of light are greatly reduced and tissue penetration depth is significantly enhanced.

In addition to structural imaging of tissue, fluorescence spectroscopy provides complementary functional information. Tissue spectroscopy is a powerful method to identify endogenous fluorescence species. The relative abundance of these species is related to tissue physiological and pathological states. Fluorescence spectroscopy has been used to characterize different tissue types such as colon, lung, cervix, and skin. ${ }^{4,5}$ In addition to tissue type characterization, spectroscopy has been used to monitor the physiological state of a

1083-3668/2005/\$22.00 (C) 2005 SPIE 


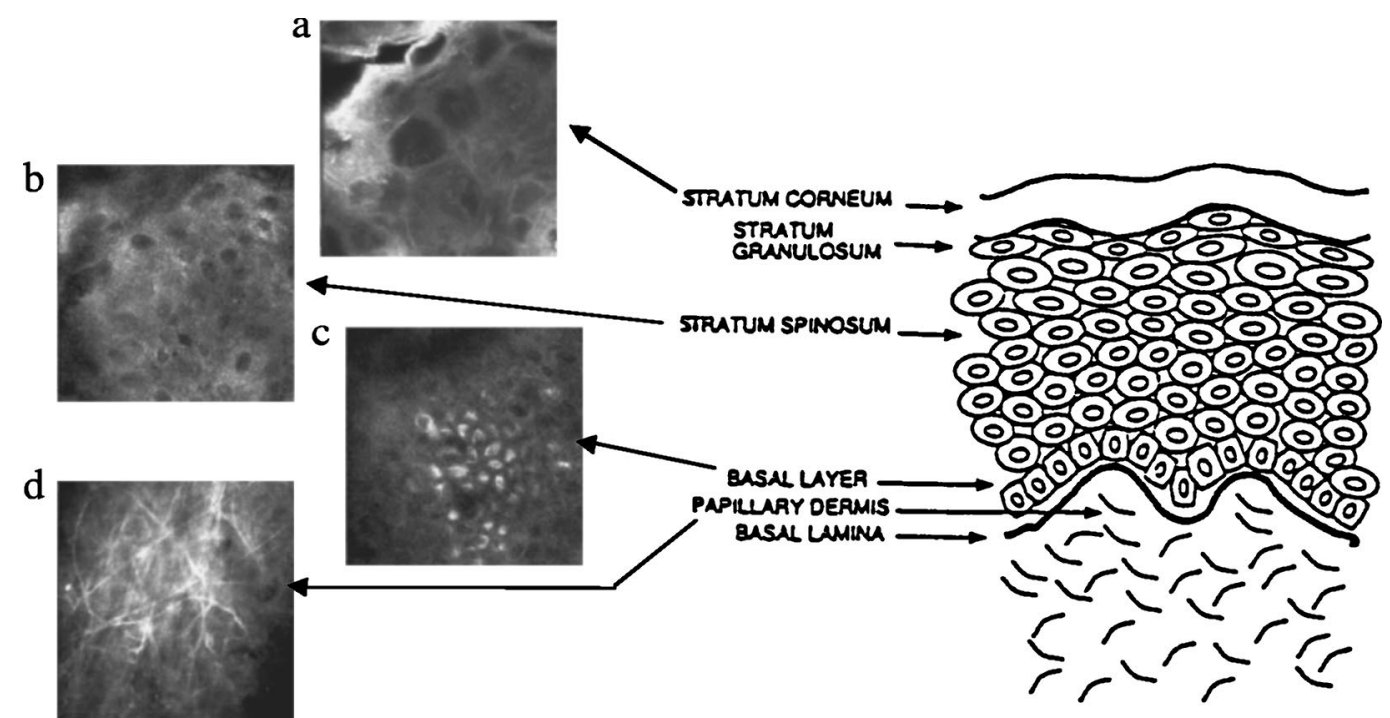

Fig. 1 Basic physiology of human skin and corresponding two-photon images of several layers: (a) stratum corneum at $5 \mu \mathrm{m}$, (b) stratum spinosum at $20 \mu \mathrm{m}$, (c) basal layer at $35 \mu \mathrm{m}$, and (d) dermis at $75 \mu \mathrm{m}$. Images taken with $2 \mathrm{~mW}$ external to specimen.

tissue. $^{6-10}$ The differences in tissue excitation spectra have been used for disease diagnosis such as distinguishing between malignant and normal tissues. ${ }^{11-14}$ Tissue spectroscopy has also been used to study the effects of aging and photoaging. ${ }^{15}$

The analysis of tissue spectroscopic information with 3-D microscopic resolution has major advantages compared with typical bulk tissue spectroscopy approaches. ${ }^{16-18}$ Previous works often examine the overall spectrum from a macroscopic sample. This overall spectrum results from a mixture of many different fluorophores. The decomposition of this "mixed" spectra into individual components based on numerical methods such as principle component analysis is often a difficult task in complex tissues. ${ }^{19-21}$ Performing spectroscopic analysis on two-photon microscopy data allows us to obtain structural information that provides additional information for spectral decomposition. Specifically, 3-D resolved spectroscopy isolates a fluorescence signal that originates from microscopic volumes that typically contain fewer independent fluorescent species. By reducing the mixing of different fluorescence species, the identity and distributions of each pure biochemical species can be more easily assayed. Spectroscopy information can be extracted from the entire volume, from a 2-D section, or even from a specific morphological structure in the sample.

While the two-photon 3-D tissue imaging and spectra recognition based on image-guided spectral analysis are applicable to the study of a variety of tissues, we focus our effort on the study of human skin. The basic physiology of human skin is seen in Fig. 1. ${ }^{22}$ Skin is comprised of two overall sections, the epidermis and dermis. The epidermis is the outermost portion of the skin and forms a protective layer to prevent loss of moisture from the body. The epidermis is comprised primarily of keratinocytes. The keratinocytes differentiate in the basal layer and migrate toward the surface of the skin. The outermost layer of the skin is the stratum corneum and is composed of dead, cornified keratinocytes. The epidermis is located above the dermis. The dermis provides struc- tural support for the epidermis and is primarily composed of a collagen and elastin matrix with a sparse population of cells such as fibroblasts.

The basic microscopic structure and bulk spectroscopic properties of human skin have been extensively studied. ${ }^{22}$ Therefore, this basic knowledge of the dermal system provides a firm basis for more in-depth spectroscopic analysis of the tissue presented in this work. Equally important, the ability to better quantify skin structure and biochemical relationships based on image and spectroscopic analysis may be useful for the diagnosis and the study of a number of skin conditions. Previous studies of skin autofluorescence have been used to study skin cancer ${ }^{23}$ and the natural aging and photoaging $^{15}$ of skin.

\section{Materials and Methods}

\subsection{Ex-Vivo Human Skin}

Ex-vivo human skin was examined in this study. The ex-vivo human skin was obtained from the expired stock of a skin bank. The specimen was stored at $-3^{\circ} \mathrm{C}$ before use. Five specimens, $1 \times 1 \mathrm{~cm}$, from the skin of two Caucasian subjects were studied. Each sample of skin was prepared by placing it in a hanging drop microscope slide containing a piece of damp sponge. The specimen was sandwiched between the coverslip and the damp sponge to maintain its moisture. Two types of samples were examined. The first type of sample was imaged in the normal orientation with the stratum corneum placed closest to the coverslip. The second type of sample was surgically exposed skin, where the exposed dermis was placed closest to the coverslip.

\subsection{Two-Photon Excitation}

A schematic of the two-photon scanning instrumentation that was used in these experiments is shown in Fig. 2. A femtosecond mode-locked Ti:sapphire laser (Tsunami, Spectra Physics, Palo Alto, California) generates the excitation light. This Ti:sapphire laser features a broad-spectrum mirror set 


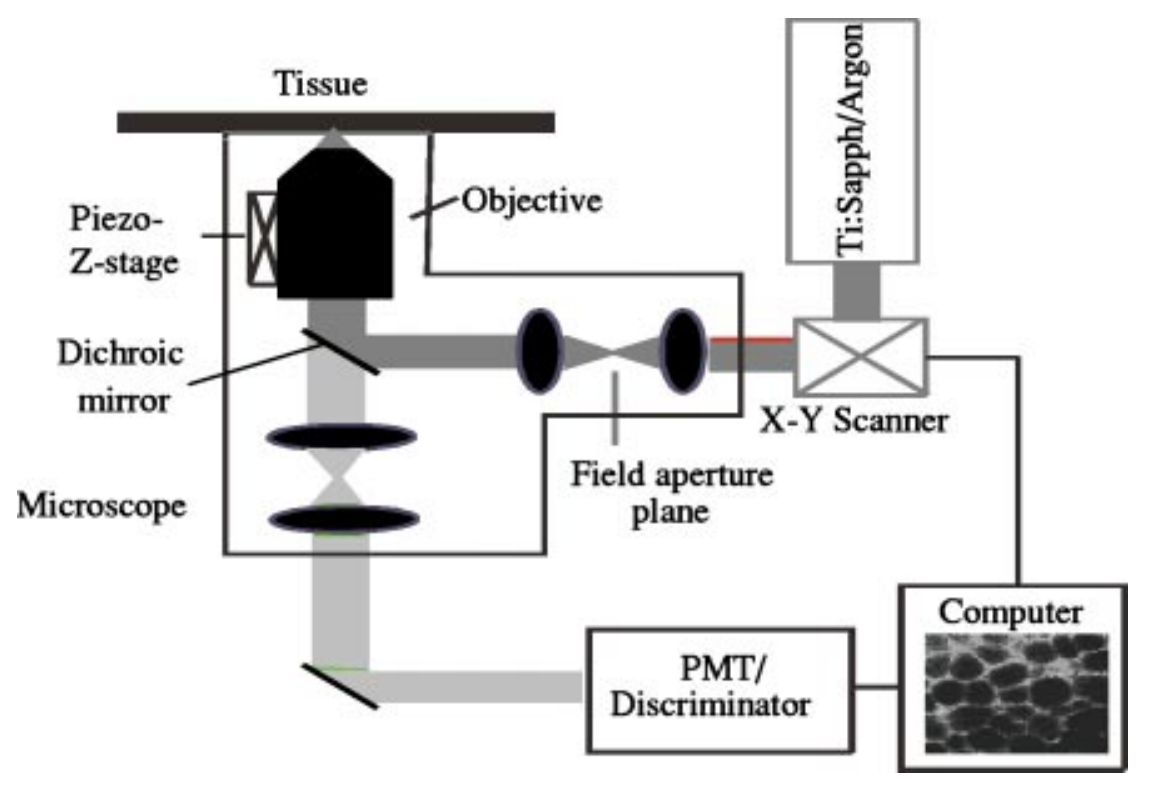

Fig. 2 Schematic of two-photon scanning microscope.

that allows the system to be conveniently tuned from 690 to $1000 \mathrm{~nm}$. A galvanometer-driven $x-y$ scanner (Cambridge Technology, Watertown, Massachusetts) is used to scan the beam through the objective and across the sample generating $256 \times 256$ pixel images. The light enters a Zeiss Axiovert 110 microscope (Zeiss Incorporated, Thornwood, New York) and is reflected into the objective through a dichroic mirror (Chroma, Brattleboro, Vermont). A piezoelectric objective translator (Physik Instrumente, Waldbronn, Germany) moves the objective in the $z$ direction, allowing for imaging at different depths. The fluorescence signal is collected by a photomultiplier tube (R7400P, Hamamatsu Corporation, Bridgewater, New Jersey) at each pixel, and a computer collects information about the number of photons at each pixel. Customized software is used to generate 3-D images. A Fluar $40 \times$, 1.25-NA oil objective (Zeiss, Thornwood, New York) was used in these experiments. The excitation power was 2 $\mathrm{mW}$ on the specimen at the coverslip. The transmission of the microscope was measured at the objective, and the excitation power was controlled so that the power incident on the sample was the same at the different excitation wavelengths. The excitation power was chosen so that there was no perceivable photodamage to the sample. This was the minimum power with which there were no morphological changes. In principle, higher power at greater depths could be used, but we have not implemented automated power control to this system.

\subsection{Emission Spectrum Resolved Imaging}

To obtain the emission spectra of the sample, a custom-made filter wheel is placed in front of the photomultiplier tube. The filters range from 375 to $600 \mathrm{~nm}$ in $25-\mathrm{nm}$ increments (filters with part number 31314, 34046, 25594, 21303, 30398, 26980, 16271, 30736, 23204, and 18106, Chroma, Rockingham, Vermont). The bandwidth of each filter is approximately $15 \mathrm{~nm}$. The uncertainty in our emission maximum determination is also $15 \mathrm{~nm}$.

\subsubsection{Image acquisition}

The size of each image acquired was $116 \times 116 \mu \mathrm{m}$ with a frame rate of $25 \mathrm{~s}$ per frame. This acquisition rate was constant throughout the volume. In the axial direction, an 80- $\mu \mathrm{m}-$ thick section of skin was imaged in $5-\mu \mathrm{m}$ step increments. Each sample of skin was imaged at three different excitation wavelengths, 730, 780, and $830 \mathrm{~nm}$, in three dimensions. For each depth within the skin, emission information was obtained by rotating the filter wheel and imaging through the various filters. This was repeated for all depths within the sample. The emission spectrum of fluorescein ${ }^{24}$ and POPOP $^{25}$ were acquired with this system, and these spectra were used to calibrate our acquired data. In addition, the intensity profile across each image was made uniform by calibrating each image to a flat-field image taken with a fluorescein solution.

\subsubsection{Analysis methods}

The two-photon images consist of a mixture of pure component responses. To extract chemically significant information from these images, we studied resolved spectra from different levels: 1. the overall bulk emission spectra of human skin, 2 . the emission spectra at individual layers within the skin, and 3. the emission spectra from specific tissue structures based on image segmentation (Fig. 3).

\section{Bulk spectrum}

To obtain the bulk emission spectrum for each excitation wavelength, the measured intensity was acquired over the entire volume. This was accomplished using two different analysis methods. The "weighted" method takes the intensity data at each pixel in the volume and sums the intensity over all pixels. This was repeated for the data imaged through each emission filter to result in the emission spectra. These steps were repeated for each of the different excitation wavelengths. The resultant spectra were then normalized. The "nonweighted" method takes the emission spectra at each pixel in the volume and normalizes each spectrum at every pixel. 


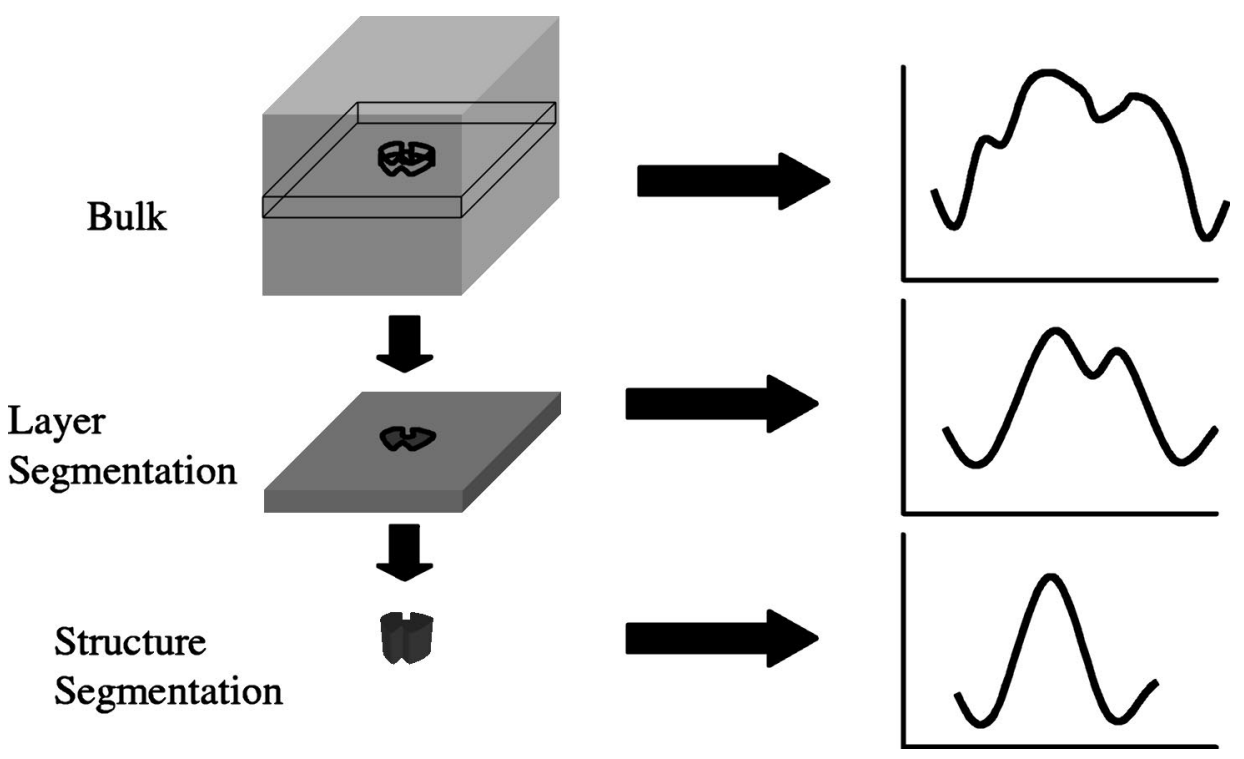

Fig. 3 Schematic of analysis methods of extracting spectral information.

These normalized intensities are then summed for each emission wavelength, which results in a single emission spectra, which is then normalized. These steps were repeated for each of the different excitation wavelengths. The nonweighted method removes the depth concentration dependence from the spectrum.

\section{Layer resolved spectrum}

The emission spectra for individual depths were obtained for the sample. We obtained the emission spectrum for four different depths that were representative of the following structures: stratum corneum, stratum spinosum, basal layer, and dermis. The individual layers were identified by morphological comparison with known histology of skin. The emission spectrum for each layer was obtained by taking the image of each layer and averaging the intensity from each pixel within the image. This imaging procedure was repeated for each emission filter to obtain the emission spectra. Each layer was excited at 730, 780, and $830 \mathrm{~nm}$. The emission spectra of surgically exposed dermis were also studied. This enabled us to further investigate the components of the dermal layer. We were also able to reconstruct the different components present in the dermis by reconstructing the overall image from each filter. This was done by imaging a $10-\mu \mathrm{m}$ section of dermis in $0.5-\mu \mathrm{m}$ increments at an excitation wavelength of $780 \mathrm{~nm}$ through each emission filter. 3-D images were then reconstructed for these emission filters.

\section{Feature resolved spectrum}

The emission spectra of different skin morphological structures can be determined. An intensity segmentation was performed on the images to resolve major structures in the skin. We select individual structures within an image and obtain the average intensity of the structure at each emission wavelength to obtain the emission spectra. By doing this, we can isolate the emission spectra associated with a particular structure and separate it from the emission spectra of the entire image.

\subsection{Fluorescence Lifetime-Resolved Imaging}

Fluorescent lifetime measurements were obtained from $e x$ vivo surgically exposed human dermis. The second harmonic generation (SHG) signal and autofluorescence from the tissue is imaged using a Zeiss Fluar $40 \times$ objective and detected by a photon counting photomultiplier tube (PMT) (R7400P, Hamamatsu, Bridgewater, New Jersey). The signal from the PMT was analyzed using a time-correlated single-photon counting card (SPC-730, Becker-Hick, ${ }^{26}$ Berlin, Germany) to build a histogram of the time of arrival of the photons at the detector. Each image contained $256 \times 256$ pixels, and each pixel contains 64 time channels spread from 0 to $17 \mathrm{~ns}$, with each bin of the time channels being 0.265 ns. Image size and resolution parameters are the same as emission spectral resolved images.

The lifetime data were fitted by iterative convolution ${ }^{27}$ using a double exponential decay convoluted with a 350-ps Gaussian full-width at half maximum (FWHM) representing the instrument response. A global fitting algorithm ${ }^{28}$ based on a least-square optimization procedure from Matlab (The Mathworks, Incorporated, Natick, Massachusetts) was used to extract two time constants from a whole image, with each decay curve at every pixel containing a variable proportion of those two time constants. Further description of the fitting algorithm and segmentation method that significantly improves the convergence speed and accuracy of the fit will be described elsewhere. ${ }^{29}$

\section{Results}

The overall emission spectrum for the entire volume of skin was obtained for three different excitation wavelengths: 730, 780 , and $830 \mathrm{~nm}$. These results can be seen in Figs. 4(a) and 4(b), which compare the weighted and nonweighted methods of generating the bulk spectrum. In the weighted method, Fig. 4(a), the bulk spectra are obtained by a photon count from 

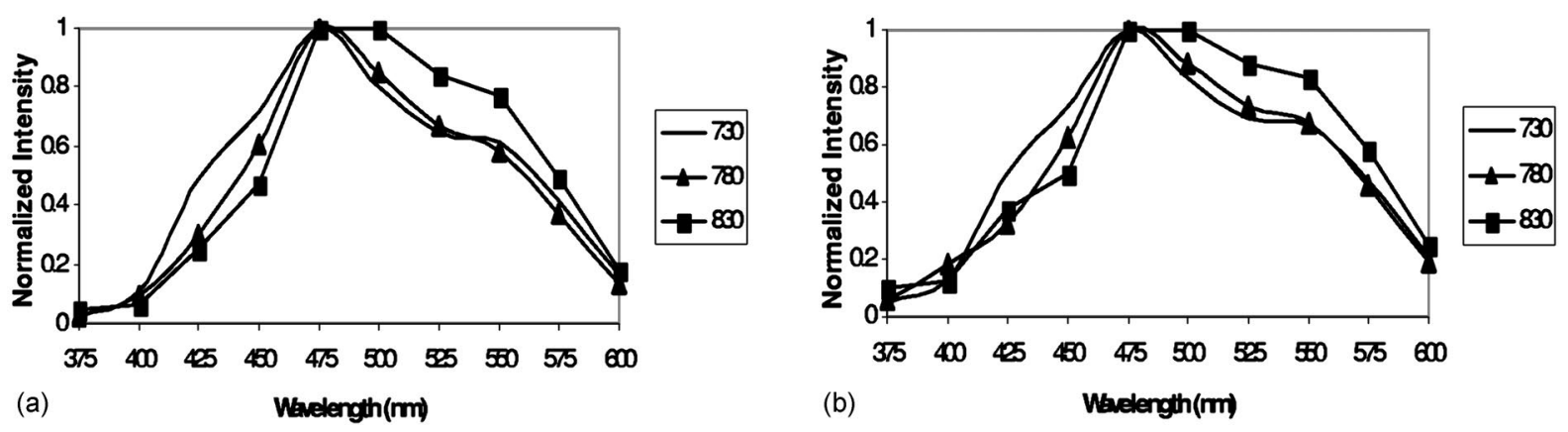

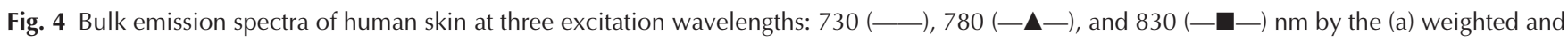
(b) nonweighted methods.

each point within the tissue and averaging them. In the nonweighted method, Fig. 4(b), the bulk spectra are obtained by weighing the different depths equally.

The emission spectrum was obtained for four different layers within the skin that were representative of the following structures: stratum corneum, stratum spinosum, basal layer, and dermis. Representative spectral data and the resulting emission spectra can be seen in Fig. 5. The emission spectra were obtained for three different excitation wavelengths: 730 , 780, and $830 \mathrm{~nm}$. Figures 5(a) through 5(d) show the same area in each layer excited at $780 \mathrm{~nm}$ and imaged using different emission filters. In each emission spectra, there are peaks at 475 and $550 \mathrm{~nm}$. When exciting at 780 and $830 \mathrm{~nm}$, one observes the appearance of two new peaks centered at 400 and 425 , respectively, that grow in intensity as one images deeper into the skin.

To better isolate the spectral contribution from dermal components without epidermal contribution, we further imaged and measured the emission spectrum from the deep dermal layer in surgically exposed skin. Emission spectrum in the deep dermis has two emission peaks at 400 and $475 \mathrm{~nm}$ at 780-nm excitation. Using feature-resolved analysis, we found that these emission peaks correspond to two morphologically distinct structures. Figure 6 presents a 3-D reconstruction of these structures with distinct spectra. The structure with a 400-nm emission maximum has a fine mesh morphology, whereas the structure with a 475-nm emission maximum has the morphology of thick ropes. We further studied the spectrum with the emission peak at $475 \mathrm{~nm}$. In the epidermis, a spectral component with emission maxima at $475 \mathrm{~nm}$ was also observed. Figure 7 shows that the spectral shapes are very different between the dermal and the epidermal components, although both components have emission maximum close to $475 \mathrm{~nm}$. We can conclude that different biochemical species are responsible for these emissions.

Figure 8(a) shows the sum of the photon counts collected using lifetime-resolved imaging. As can be seen in the graphs in Figs. 8(b) and 8(c), each pixel of the image contains the fluorescence decay information collected in this pixel. These two graphs contain five decay curves representative of the areas highlighted in Fig. 8(a).

The lifetime image has been fitted using the following equation, where $I_{i}$ is the intensity as a function of time in pixel $i$ and $G(t)$ the Gaussian instrument response:

$$
\begin{aligned}
I_{i}(t)= & \int_{0}^{t} G(t-T) \cdot c_{2 i+1}\left[c_{2 i+2} \exp \left(-\frac{T}{c_{1}}\right)+(1\right. \\
& \left.\left.-c_{2 i+2}\right) \exp \left(-\frac{T}{c_{2}}\right)\right] d T .
\end{aligned}
$$

The coefficients $c_{1}$ and $c_{2}$ are the two time constants extracted from the image and were obtained as $47 \mathrm{ps}$ and $2.6 \mathrm{~ns}$. The coefficient $c_{2 i+1}$ represents the initial intensity in each pixel $i$, while $c_{2 i+2}$ corresponds to the ratio of the two decays in this same pixel. Figure 9(a) is a map of the value of these coefficients $c_{2 i+2}$ obtained from the global curve fitting. Figure 9(b) shows four decay curves with their corresponding fits. The insert shows the residuals (difference between fitting curve and experimental data) for those four curves. The largest error on the fits is on the order of $10 \%$ and occurs around the time of the excitation. The use of an experimentally recorded instrument response might help to better fit the very fast growth and decay of the signal. Figure 9(c) is a complete map of the sum of the absolute value of the residuals normalized by the total intensity in each pixel. The absence of structure in this image shows that the optimization is successful both in regions of high or low autofluorescence.

\section{Discussion}

The bulk spectrum obtained in this experiment can be evaluated as either weighted or nonweighted. In principle, the weighted method is more similar to traditional tissue spectroscopy ${ }^{5,16}$ in which the spectrum is modified by both the tissue scattering and absorption of the excitation light and the emission light, resulting in giving the spectroscopic features from the surface of the tissue a greater contribution. The degree of weighting depends on tissue type, wavelengths, locale, and morphology and is, in general, not very well quantified. In contrast, the nonweighted method weighs the contribution from each depth more equally and can provide a better estimate of the true emission spectrum. The difference between these two methods can be seen by comparing Figs. 4(a) and 4(b). We found that the weighted and nonweighted measurement is not greatly different for human skin down to $150 \mu \mathrm{m}$. The main observable difference is that the contribution of the fluorophore emitting at $550 \mathrm{~nm}$ is greater in the nonweighted versus the weighted method. This indicates that the scattering 
(a)
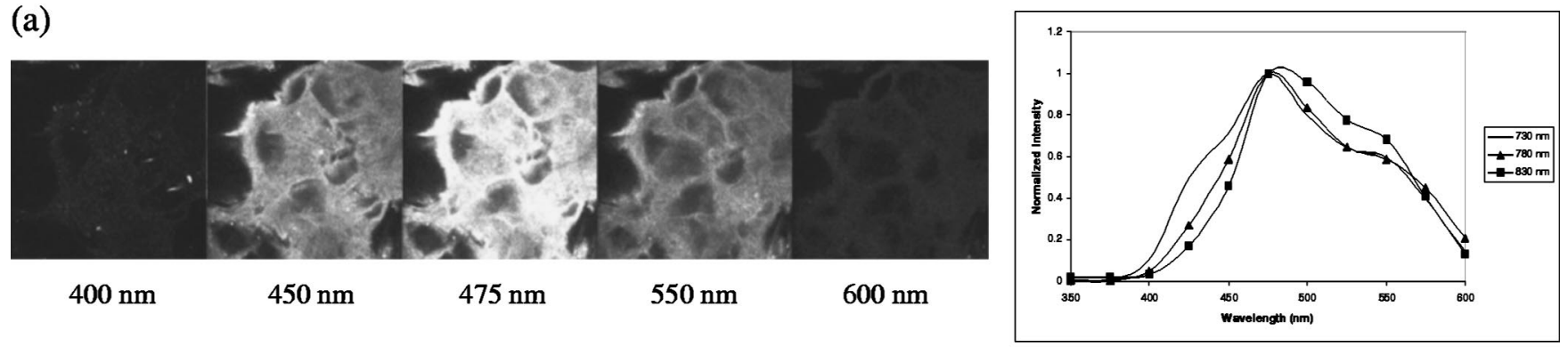

(b)
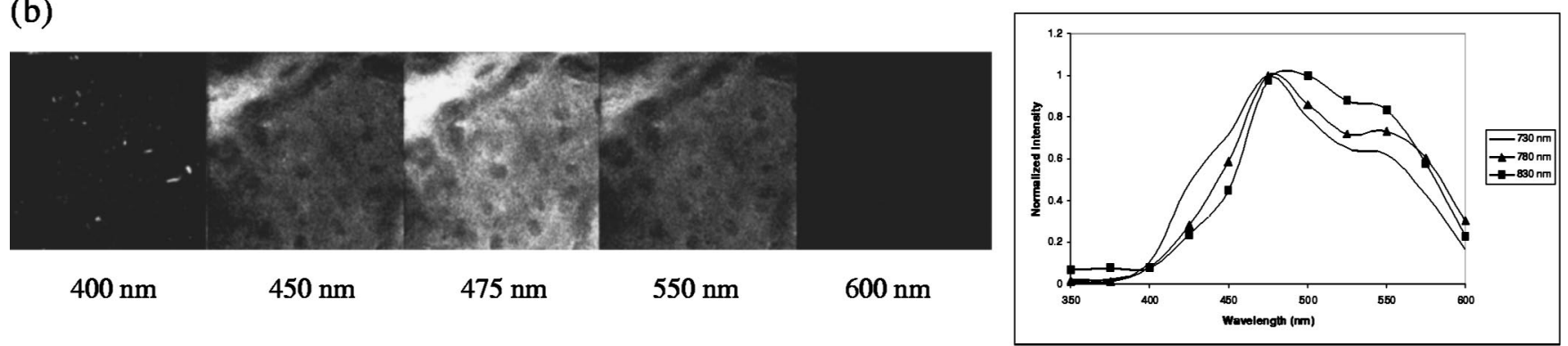

(c)
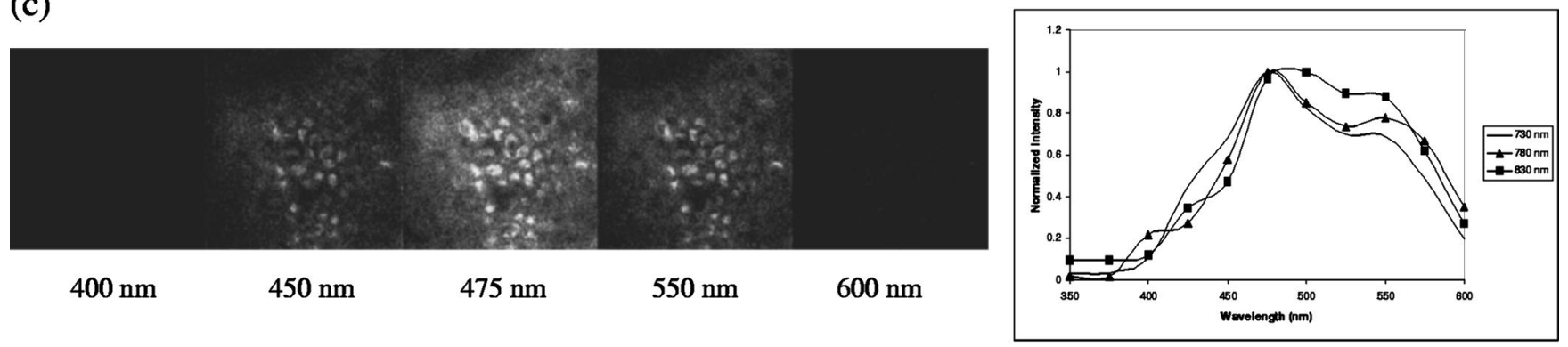

(d)
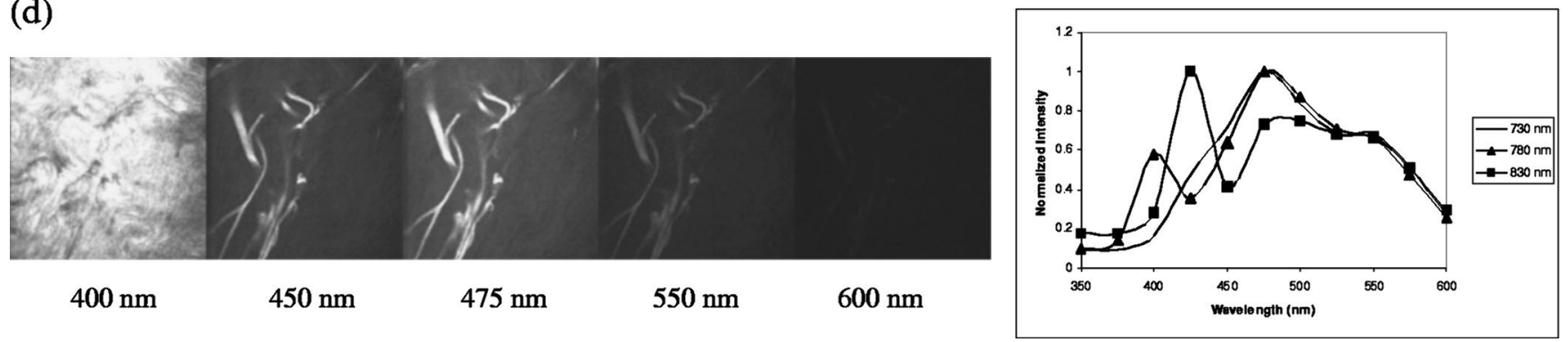

Fig. 5 Two-photon images of various skin layers obtained after excitation at $780 \mathrm{~nm}$ and observed through different emission filters. The emission spectra of each layer for different excitation wavelengths are also shown (a) stratum corneum, (b) stratum spinosum, (c) basal layer, and (d) dermis. Each layer is excited at three different excitation wavelengths: $730(-), 780(-\mathbf{\Delta}-)$, and $830(-\square) \mathrm{nm}$. 


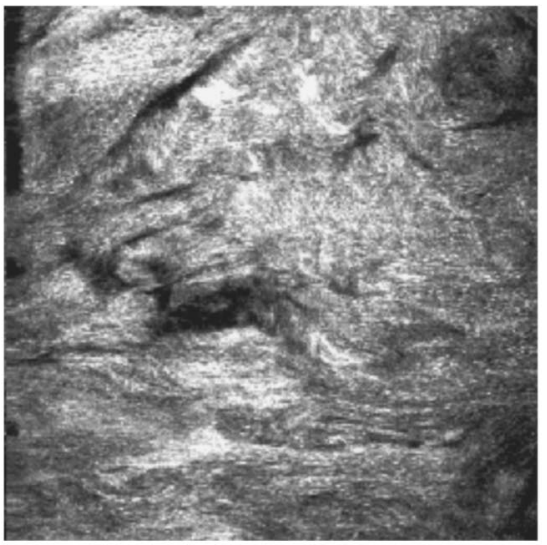

(a)

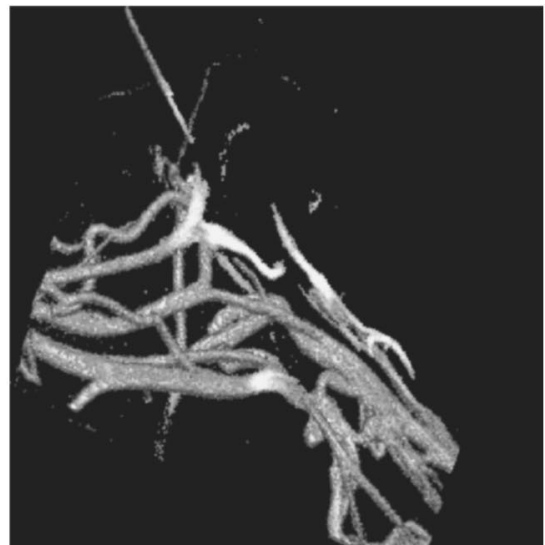

(b)

Fig. 6 Dermis 3-D reconstruction at emission wavelengths of (a) 400 and (b) $475 \mathrm{~nm}$.

effect does not significantly compromise spectral measurement for epidermal and shallow dermal structures. In addition to causing the bulk spectrum to be surface weighted, scattering may further result in a progressive red shift of the spectral peaks for a given biochemical specie at increasing depth. This effect is due to the higher scattering probability for shorter wavelength light. However, the results, as represented by Fig. 5 , show that this effect is not observed. The emission spectral peaks of all autofluorescence species, such as $\mathrm{NADH}$, remain constant as a function of depth for the skin layers examined in this report. This evidence further supports the conclusion that multiple scattering has minimal effect in spectroscopic measurement of skin down to a depth of 100 to $200 \mu \mathrm{m}$.

It is apparent that the interpretation of the bulk spectrum is difficult, and it is nearly impossible to identify all the chemical components present within the skin or determine their concentration. To extract that information, a layer segmentation was more successful. We were able to determine the biochemical factors that contribute to most of the autofluoresence that we see in skin. This was accomplished by analyzing the emission spectra of the different layers within the skin. The four different layers each have peaks at 475 and $550 \mathrm{~nm}$. The 475-nm peak corresponds to known NAD(P)H emission spectra, ${ }^{30}$ and $\operatorname{NAD}(\mathrm{P}) \mathrm{H}$ is known to be present in cells and therefore is present in the top three layers.

The 550-nm peak is associated with the presence of melanin in the tissue. The peak at $550 \mathrm{~nm}$ is seen to be increasing as a function of depth, with the highest normalized intensity occurring within the basal layer. The increase in peak intensity as we move from the stratum corneum into the basal layer is as expected for melanin. In addition, comparison of the ratio of the peaks at 475 to $550 \mathrm{~nm}$ shows that the relative concentrations of the melanin are increasing as compared to $\mathrm{NAD}(\mathrm{P}) \mathrm{H}$ as we move deeper into the skin. This higher distribution of melanin in the deeper epidermal layer is also consistent with the observation that the $550-\mathrm{nm}$ peak is more pronounced in the nonweighted bulk spectrum as compared with the weighted bulk spectrum.

In Figs. 5(a) and 5(b), a shoulder can be seen at $425 \mathrm{~nm}$. This feature only occurs within the epidermis and under excitation at $730 \mathrm{~nm}$. The location of this fluorescent species is consistent with the distribution of tryptophan within the keratinocytes. Although tryptophan is typically excited at approximately $290 \mathrm{~nm}$ with one-photon, ${ }^{31,32}$ it is plausible that we

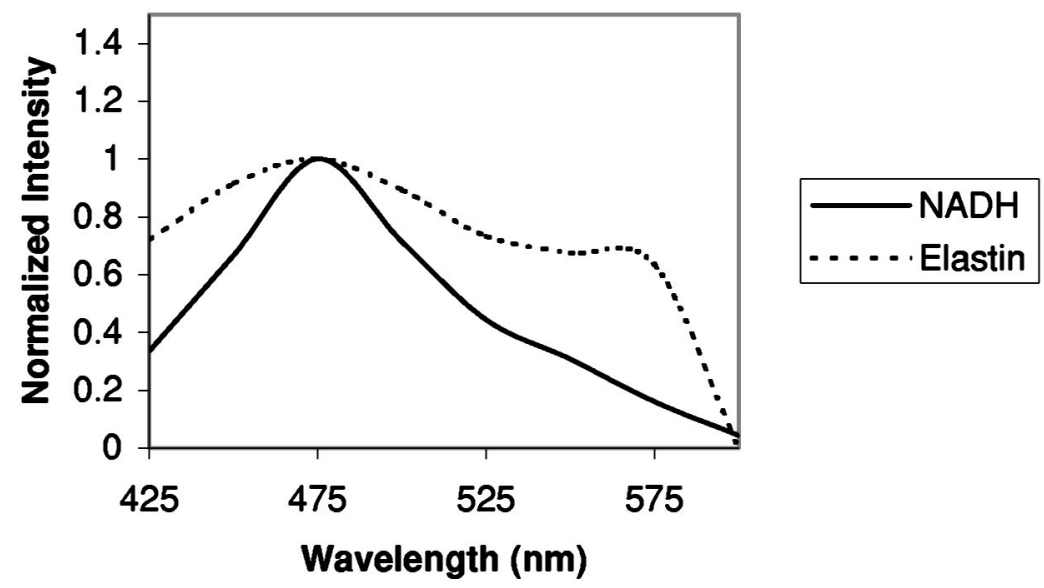

Fig. 7 Emission spectra of reversed dermis from resolved spectrum based on tissue morphology. 

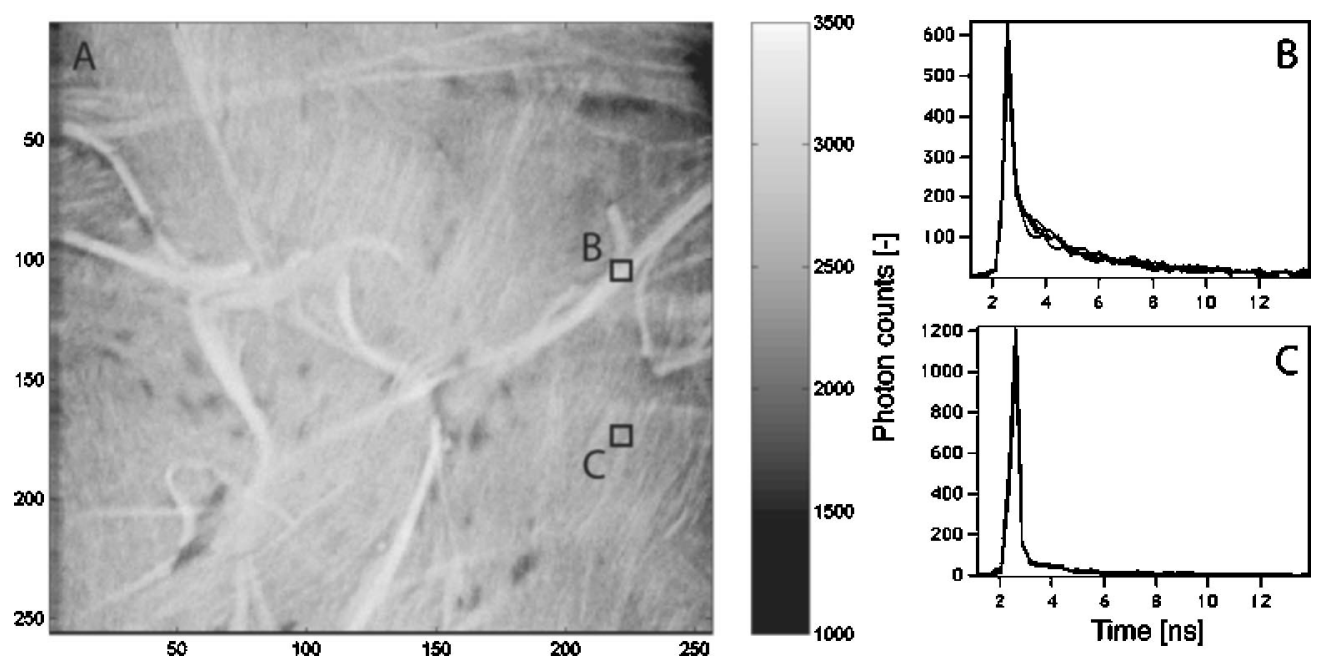

Fig. 8 SHG and fluorescence decay collected by time-correlated single-photon counting. (a) Total number of photon counts collected in each pixel, and (b) and (c) show five typical emission decays from the regions highlighted in (a).

may still pick up the tail end of the emission spectra of tryptophan. We only see a slight peak at $425 \mathrm{~nm}$ because our optics do not pass wavelengths below $350 \mathrm{~nm}$.

Finally, we found the spectrum of the dermis to be quite interesting. There are two distinct peaks within this layer. The first peak is located at about $400 \mathrm{~nm}$ when excited at $780 \mathrm{~nm}$, and shifts to $425 \mathrm{~nm}$ when excited at $830 \mathrm{~nm}$. There is a shift in the peak emission as the excitation wavelength shifts. Second harmonic generation is a nonlinear process where two photons combine together to generate a new photon. The energy of the photon created is the sum of the energies of the incoming photons. In the present case, the observation that the emission wavelength is approximately at half of the laser frequency is consistent with the occurrence of SHG in the sample. The slight offsets of the SHG signals from the expected wavelengths are due to wavelength measurement uncertainty, since relatively broad bandpass filters were used. Collagen is well known to produce SHG in biological samples $^{33}$ and is responsible for this signal in our emission spectra. The second emission peak at $475 \mathrm{~nm}$ is due to autofluorescence as indicated from the significantly broader spectral width. It is known from previous studies that both $\mathrm{NAD}(\mathrm{P}) \mathrm{H}$ and elastin emit into this band. ${ }^{30,34}$ Therefore, the observed signal may arise from either cellular or extracellular matrix sources. To resolve this ambiguity, we performed a feature-based segmentation to further resolve the emission spectra. Regions corresponding to $\mathrm{NAD}(\mathrm{P}) \mathrm{H}$ and elastin excited at $780 \mathrm{~nm}$ were selected, and the emission spectra associated with these regions were generated and presented in Fig. 7. By studying these spectra, regions that we believe to be corresponding to elastin appear to have a broader peak, while regions corresponding to $\mathrm{NAD}(\mathrm{P}) \mathrm{H}$ contain a narrower peak that we think may be $\mathrm{NAD}(\mathrm{P}) \mathrm{H}$ in the fibroblasts.

We used lifetime resolved imaging to further support our identification of luminescence species in the dermis. This method further allows us to precisely determine the relative abundance of these species. The decay curve of the coarse fibrous structure observed in the intensity image of Fig. 8 has
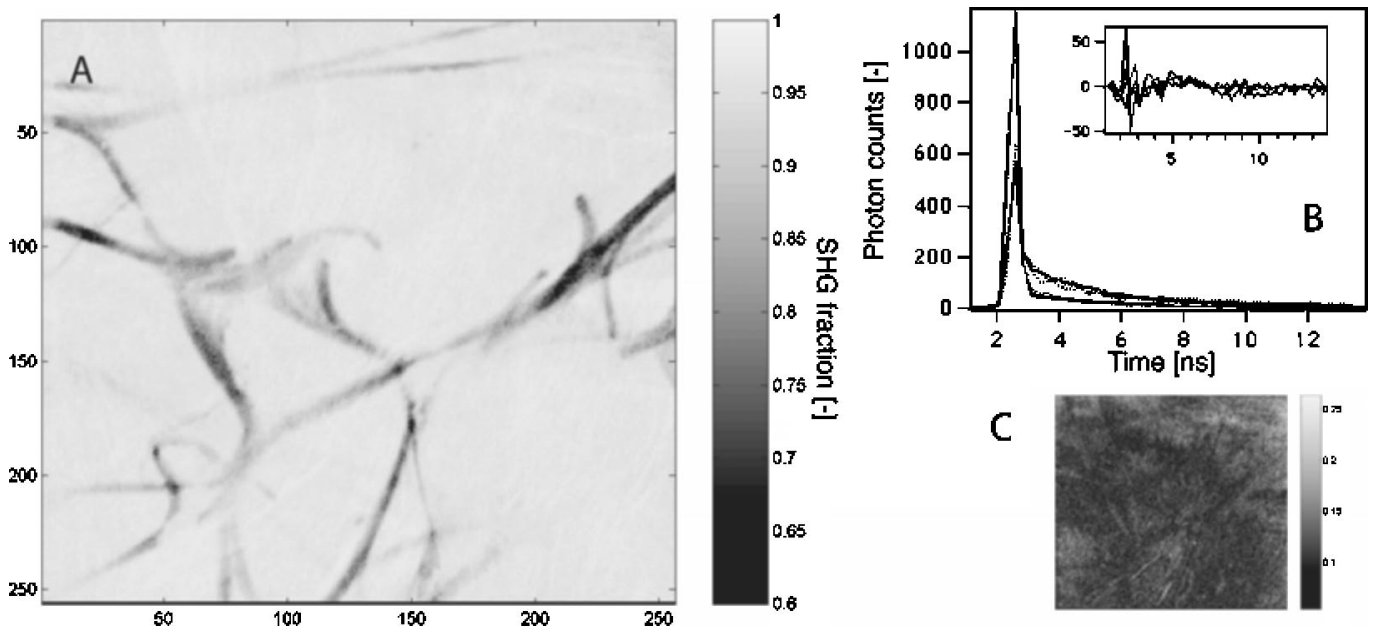

Fig. 9 (a) The ratio of SHG emission versus autofluorescence obtained by global fitting of the lifetime image. (b) Four experimental decays (dotted lines) and their corresponding fits (solid lines). (c) The image of the sum of the residuals. 
Table 1 Summary of autofluorescent components. Elastin is located in the dermis. Collagen is due to second harmonics, and "melanin" can also mean a biochemical component associated with melanin.

\begin{tabular}{lccc} 
& \multicolumn{3}{c}{ Excitation wavelength $(\mathrm{nm})$} \\
\cline { 2 - 4 } $\begin{array}{l}\text { Peak emission } \\
\text { wavelength }(\mathrm{nm})\end{array}$ & 730 & 780 & 830 \\
\hline 400 & & Collagen & \\
425 & Tryptophan & & Collagen \\
475 & $\mathrm{NADH}$ & $\mathrm{NADH} /$ elastin & $\mathrm{NADH} /$ elastin \\
550 & Melanin & Melanin & Melanin \\
\hline
\end{tabular}

a longer fluorescent lifetime [Fig. 8(b)] compared to the finer mesh background of the image, which shows a spike in the short fluorescence lifetime component but very limited long lifetime emission [Fig. 8(c)]. The global fitting algorithm helps to further dissociate these two behaviors. The short lifetime of $47 \mathrm{ps}$ (much shorter than the instrument response of $350 \mathrm{ps}$ ) is consistent with light arising from second harmonic generation, which is the signature of collagen in the sample. An independent control experiment further confirms that the scattered light intensity is negligible (data not shown).

The longer lifetime component of the emitted light measured is consistent with autofluorescence from elastin. ${ }^{35}$ However, one should note that the limited photon counts collected from the autofluorescent and the presence of the strong peak due to SHG does not allow the resolution of the lifetimes of more than a single fluorescent species. Therefore, the fluorescent signal may originate from other endogenous fluorophores in addition to elastin.

The global fitting technique allows us to map the contribution from both components in the image. The distribution of SHG signal from collagen and autofluorescence signal from elastin corresponds well with the expected dermis structure as known from histology and from our emission wavelength data. It is also interesting to notice that the distribution of the SHG is dominant throughout the dermis, contributing at least $60 \%$ of the total intensity. This indicates that the distribution of collagen is ubiquitous in dermis in micron length scale. Elastin coexists with collagen in coarse fibers with approximately equal intensity contributions.

\section{Conclusions}

We apply nonlinear microscopic imaging and spectroscopy to study the biochemical identities of luminescence species and their distribution in ex-vivo human skin. Since the distributions of chromophores in skin are not uniform and vary as a function of location, we apply a morphologically guided spectral analysis method to resolve the constituent luminescence components associated with different skin morphological structures. By linking the spectroscopic information to tissue morphological structures, we successfully identify the luminescence components in human skin as summarized in Table 1. We find that morphologically guided spectral analysis is a very powerful general approach for tissue biochemical analysis with applications beyond dermatological studies.
Sample preparation procedures, such as from excision, storage, and fixation, may affect the spectral properties of ex-vivo tissue. In the future for clinical diagnosis, additional experiments should be done to determine the skin spectra of patients in vivo. In-vivo study will further allow us to address spectral differences due to age, race, and skin pigmentation of the patients. We do not expect the spectra shape and the autofluorescence species in the skin to be significantly different between future in-vivo studies as compared to this ex-vivo work. However, there may be variations observed for the relative concentrations of some biochemical components due to protein degradation or metabolic differences. This study serves to identify the major autofluorescent components in the skin and forms the basis for future in-vivo skin spectroscopic studies and clinical examinations. In addition to the study of the dermal system, this type of spectroscopic study should also find applications in the diagnosis of physiological and pathological states of other tissues. However, these opportunities are currently limited by the lack of clinical portability of typical two-photon microscopes that may be overcome by the future development of a multiphoton endoscope.

\section{Acknowledgment}

This work is supported by Unilever Research U.S., Incorporated, American Cancer Society (RPG-98-058-01-CCE), and National Institute of Health (R33 CA091354-01A1). S. Pelet thanks the support from the Swiss National Science Foundation.

\section{References}

1. M. Goppert-Mayer, "Uber elementarakte mit zwei quantensprungen," Ann. Phys. 9, 273-294 (1931).

2. W. Denk, J. H. Strickler, and W. W. Webb, "Two-photon laser scanning fluorescence microscopy," Science 248, 73-76 (1990).

3. C. Buehler, K. H. Kim, C. Y. Dong, B. R. Masters, and P. T. C. So, "Innovations in two-photon deep tissue microscopy," IEEE Eng. Med. Biol. 18, 23-30 (1999).

4. A. M. K. Nilsson, D. Heinrich, J. Olajos, and S. Andersson-Engels, "Near infrared diffuse reflection and laser-induced fluorescence spectroscopy for myocardial tissue characterization," Spectrochim. Acta, Part A 53, 1901-1912 (1997).

5. H. Zeng, C. MacAulay, D. I. McLean, and B. Palcic, "Spectroscopic and microscopic characteristics of human skin autofluorescence emission," Photochem. Photobiol. 61, 639-645 (1995).

6. R. M. Cothren, R. Richards-Kortum, M. Sivak, M. Fitzmaurice, R. Rava, G. Boyce, M. Doxtader, R. Blackman, T. Ivanc, G. Hayes, M. S. Feld, and R. E. Petras, "Gastrointentinal tissue diagnostic by laserinduced fluorescence spectroscopy at endoscopy," Gastrointest. Endosc. 36, 105-111 (1990).

7. R. Richards-Kortum, R. P. Rava, M. Fitzmaurice, L. Tong, N. B. Ratliff, J. R. Kramer, and M. S. Feld, "A one-layer model of laser induced fluorescence for diagnosis of diseases in human tissue: application to human tissue," IEEE Trans. Biomed. Eng. 36, 12221232 (1989).

8. R. Richards-Kortum, R. P. Rava, R. E. Petras, M. Fitzmaurice, M. Sivak, and M. S. Feld, "Spectroscopic diagnosis of colonic dysplasia," Photochem. Photobiol. 53, 777-786 (1991).

9. S. P. Schilders and M. Gu, "Three-dimensional autofluorescence spectroscopy of rat skeletal muscle tissue under two-photon excitation," Appl. Opt. 38, 720-723 (1999).

10. M. G. Xu, E. D. Williams, E. W. Thompson, and M. Gu, "Effect of handling and fixation processes on fluorescence spectroscopy of mouse skeletal muscles under two-photon excitation," Appl. Opt. 39, 6312-6317 (2000).

11. R. Glasgold, M. Glasgold, H. Savage, J. Pinto, R. Alfano, and S. Schantz, "Tissue autofluorescence as an intermediate endpoint in NMBA-induced esophageal carcinogenesis," Cancer Lett. 82, 33-41 (1994). 
12. S. P. Schantz, H. E. Savage, P. Sacks, and R. R. Alfano, "Native cellular fluorescence and its application to cancer prevention," Environ. Health Perspect. 105, 941-944 (1997).

13. K. Licha, B. Riefke, V. Ntziachristos, A. Becker, B. Chance, and W. Semmler, "Hydrophilic cyanine dyes as contrast agents for nearinfrared tumor imaging: Synthesis, photophysical properties and spectroscopic in vivo characterization," Photochem. Photobiol. 72, 392-398 (2000).

14. B. Beauvoit and B. Chance, "Time-resolved spectroscopy of mitochondria, cells, and tissues under normal and pathological conditions," Mol. Cell. Biochem. 184, 445-455 (1998).

15. N. Kollias, R. Gillies, M. Moran, I. E. Kochevar, and R. R. Anderson, "Endogenous skin fluorescence includes bands that may serve as quantitative markers of aging and photoaging," J. Invest. Dermatol. 111, 776-780 (1998).

16. R. Na, I. M. Stender, L. Ma, and H. C. Wulf, "Autofluorescence spectrum of skin: component bands and body site variations," Skin Res. Technol. 6, 112-117 (2000).

17. H. Zeng, C. MacAulay, B. Palcic, and D. I. McLean, "A computerized autofluorescence and diffuse reflectance spectroanalyser system for in vivo skin studies," Phys. Med. Biol. 38, 231-240 (1993).

18. R. R. Alfano, D. Tata, J. Cordero, P. Tomashefsky, F. Longo, and M. Alfano, "Laser induced fluorescence spectroscopy from native cancerous and normal tissues," Inst. Electr. Electron. Eng. J. Quant. Elect. QE-20, 1507-1511 (1984).

19. C. Y. Wang, C. T. Chen, C. P. Chiang, S. T. Young, S. N. Chow, and H. K. Chiang, "A probability-based multivariate statistical algorithm for autofluorescence spectroscopic identification of oral carcinogenesis," Photochem. Photobiol. 69(4), 471-477 (1999).

20. H. Tsurui, H. Nishimura, S. Hattori, S. Hirose, and K. Okumura, "Seven-color fluorescence imaging of tissue samples based on Fourier spectroscopy and singular value decomposition," J. Histochem. Cytochem. 48(5), 653-662 (2000).

21. A. J. Andrew and T. M. Hancewicz, "Rapid analysis of Raman image data using two-way multivariate curve resolution," Appl. Spectrosc. 52(6), 797-807 (1998).

22. B. R. Masters, P. T. C. So, and E. Gratton, "Multiphoton excitation fluorescence microscopy and spectroscopy of in vivo human skin," Biophys. J. 72, 2405-2412 (1997).
23. J. Hewett, T. McKechnie, W. Sibbett, J. Ferguson, C. Clark, and M. Padgett, "Fluorescence detection of superficial skin cancers," J. Mod. Opt. 47, 2021-2027 (2000).

24. R. P. Haugland, Handbook of Fluorescent Probes and Research Products, 9th ed., Molecular Probes, Inc., Eugene, OR (2002).

25. I. B. Berlman, Handbook of Fluorescence Spectra of Aromatic Molecules, Academic Press, New York (1971).

26. "SPC-300 through SPC-730 Operating Manual," Becker \& Hickl $\mathrm{GmbH}$, see http://www.becker-hickl.de.

27. A. E. McKinnon, A. G. Szabo, and D. R. Miller, "The deconvolution of photoluminescence data," J. Chem. Phys. 81, 1564-1570 (1977).

28. P. J. Verveer, A. Squire, and P. I. H. Bastiaens, "Global analysis of fluorescence lifetime imaging microscopy data," Biophys. J. 78, 2127-2137 (2000).

29. S. Pelet, M. J. R. Previte, L. H. Laiho, and P. T. C. So, "A fast global fitting algorithm for fluorescence lifetime imaging microscopy based on image segmentation," Biophys. J. 87, 2807-2817 (2004).

30. B. Chance and B. Thorell, "Localization and kinetics of reduced pyridine nucleotide in living cells by microfluorometry," J. Biol. Chem. 234, 3044-3050 (1959).

31. S. Underfriend, Fluorescence Assay in Biology and Medicine, Vol. 1, Academic Press, New York (1962).

32. B. Brancaleon, G. Lin, and N. Kollias, "The in vivo fluorescence of tryptophan moieties in human skin increases with UV exposure and is a marker for epidermal proliferation," J. Invest. Dermatol. 113, 977982 (1999).

33. E. Georgiou, T. Theodossiou, V. Hovhannisyan, K. Politopoulos, G. S. Rapti, and D. Yova, "Second and third optical harmonic generation in type I collagen, by nanosecond laser irradiation, over a broad spectral region," Opt. Commun. 176, 253-260 (2000).

34. K. Konig and I. Riemann, "High-resolution multiphoton tomography of human skin with subcellular spatial resolution and picosecond time resolution," J. Biomed. Opt. 8, 432-439 (2003).

35. J. M. I. Maarek, L. Marcu, W. J. Snyder, and W. S. Grundfest, "Time resolved fluorescence spectra of arterial fluorescent compounds: reconstitution with the Laguerre expansion technique," Photochem. Photobiol. 72, 78-187 (2000). 